\title{
Results from the Commissioning of the ATLAS Pixel Detector with Cosmics Data
}

\author{
Jens Weingarten, on behalf of the ATLAS Pixel Detector Collaboration
}

\begin{abstract}
The ATLAS pixel detector consists of three barrel layers and three disk-layers in the endcaps, comprising a total of 80 million pixel cells. Commissioning for the pixel detector has started in spring 2008. A representative selection of commissioning results is given.
\end{abstract}

Key words: ATLAS, Pixel, Commissioning

\section{Introduction}

The ATLAS pixel detector consists of 1744 individual modules, each comprising 46080 pixels of $50 \times 400 \mu \mathrm{m}^{2}$ [1]. The connection between modules and off-detector electronics is made using a custom optical link.

The detector was installed in the center of ATLAS in June 2007.

\section{Commissioning in 2008}

Commissioning of the pixel detector started in April 2008 when the electrical, optical, and cooling connections were finished. It was soon interrupted by a failure of the cooling system, to be restarted only in August with the adjustment of the operational parameters of the optical link followed by verification tests to determine the largest possible sample of modules to be included in a combined ATLAS data taking run. After the LHC incident the commissioning program was extended until the end of 2008 to refine the parameters of the optical links as well as to improve the module performance and to obtain a complete set of calibrations needed for offline data analysis.

\section{Calibration Results}

\section{Optical link tuning}

An automated tuning procedure is used to determine the optimal set of parameters in on-detector laser power, off-detector threshold and data sampling phase. Using this algorithm $96 \%$ of all links could be adjusted, the remaining ones needing some manual fine-tuning due to effects like large spread of laser power across one laser array.

\section{Threshold and noise}

The discriminator threshold for each pixel is adjusted to $4000 \mathrm{e}^{-}$. Figure 1 shows the threshold and noise distributions for $94 \%$ of all pixels, showing a threshold non-uniformity of $1 \%$. The noise distribution peaks at $165 \mathrm{e}^{-}$.

\section{Charge measurement}

The charge measurement, using the time-over-threshold (TOT)
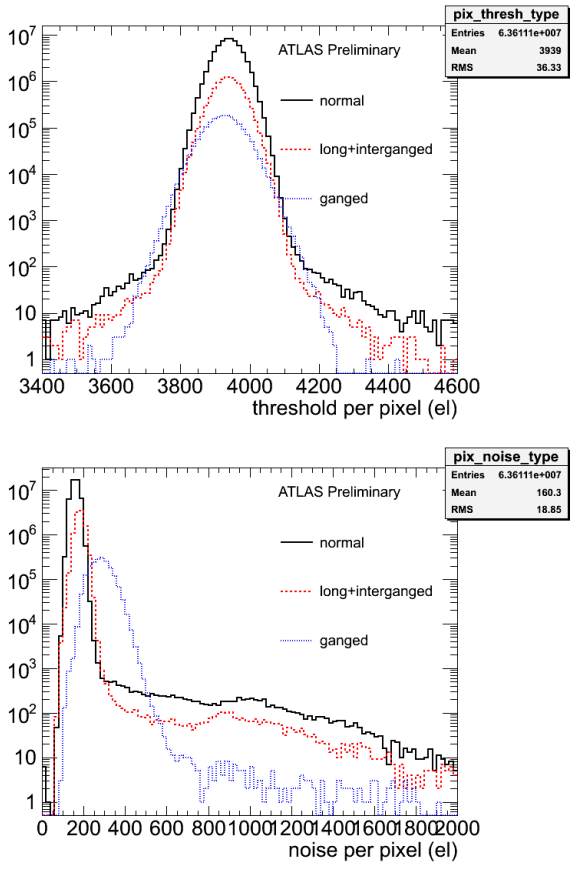

Figure 1: Top: Distribution of discriminator thresholds over 75 million pixels with an RMS of $40 \mathrm{e}^{-}$. Bottom: Distribution of single channel noise over 75 million pixels, peaking at $165 \mathrm{e}^{-}$.

method, is adjusted for every pixel to yield a TOT of $30 \mathrm{LHC}$ bunch-crossing units (BX) for a charge deposition of $20 \mathrm{ke}^{-}$. Figure 2 shows the TOT distribution for $94 \%$ of all pixels, displaying a non-uniformity of only $2 \%$ in TOT.

\section{Cosmic Data Taking}

In the combined ATLAS and Inner Detector stand-alone cosmic runs a total of 450000 tracks were recorded with hits in the pixel detector. During the data taking $96 \%$ of all pixel modules were operational. The hit efficiency of the three barrel layers was measured to be around $99.8 \%$, excluding known bad modules (see Figure 4). After masking noisy pixels accounting for less than $10^{-4}$ of all pixels an occupancy due to noise hits below 


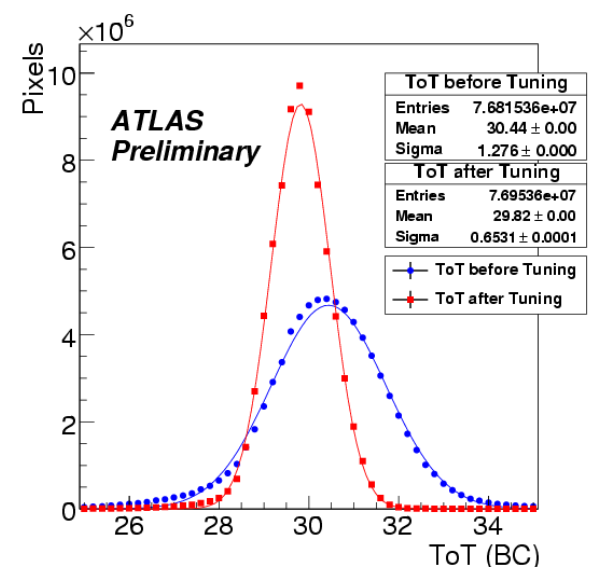

Figure 2: Distribution of TOT values over 75 million pixels with an RMS of $0.65 \mathrm{BX}$.

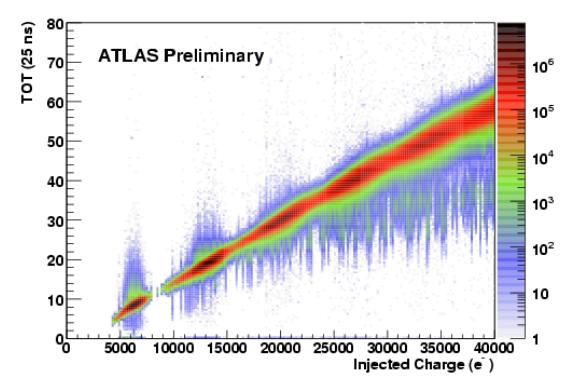

Figure 3: Correlation of TOT with injected charge. Color-coded is the number of pixels.

$2 \times 10^{-10}$ was measured, as shown in Figure 5.

The data was used for alignment of the pixel detector, reducing the width of the distribution of track residuals for clusters with two hit pixels to $23.4 \mu \mathrm{m}$ in the $50 \mu \mathrm{m}$ pixel direction using charge interpolation between the clustered pixels (see Figure 6). Using the TOT calibration (Figure 3 ) the reconstructed charge for clusters associated to tracks was measured to be within $7 \%$ of the expected value.

\section{Conclusions}

The commissioning of the ATLAS pixel detector started in august 2008, achieving basic data taking readiness in time for the first LHC beams in september. After the LHC incident the commissioning program was extended until the end of 2008, concentrating on improving the detector efficiency through improvements in software and to refining the tuning of the parameters of the optical links. The commissioning phase was also used to successfully train about 40 non-expert detector operators for continued shift operation.

\section{References}

[1] G. Aad et al., ATLAS Pixel Detector Electronics and Sensors. JINST 3 $\mathrm{P} 07007,2008$

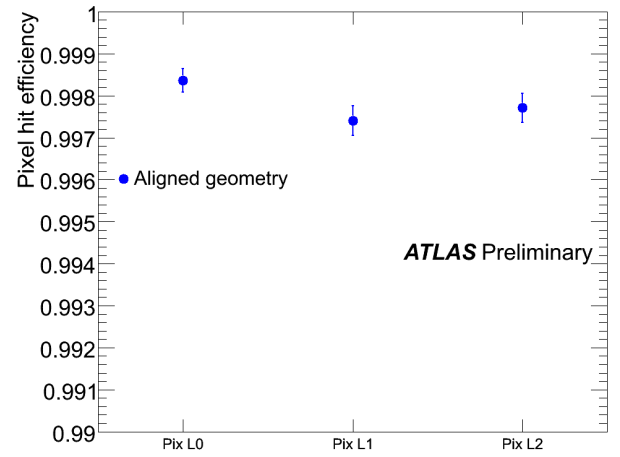

Figure 4: Hit efficiency of the three barrel layers measured using cosmic tracks. The hit efficiency is around $99.8 \%$.

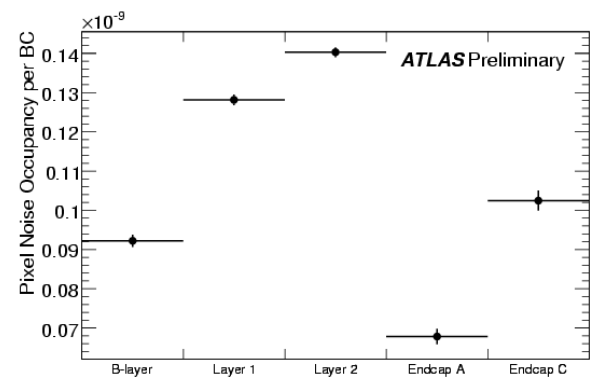

Figure 5: Noise occupancy after masking a fraction of about $10^{-4}$ pixels due to noise.

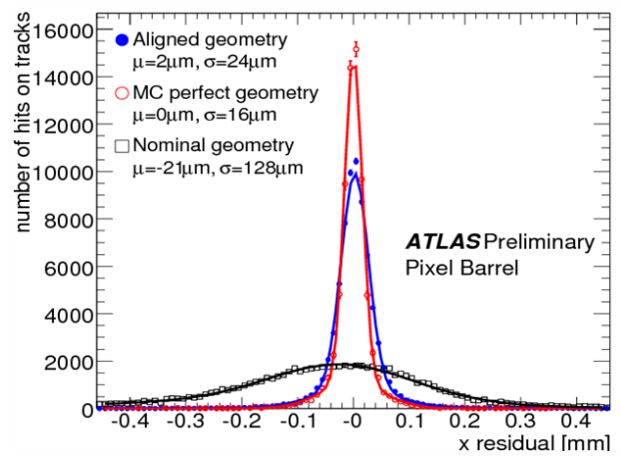

Figure 6: Comparison of track residual distributions for the nominal and the simulated perfect geometry, as well as with the aligned geometry as calculated using the cosmics data. The width of the latter distribution is $24 \mu \mathrm{m}$. 